\title{
Cardinality Constrained Portfolio Optimization Using bee Colony Algorithm (Case Study: Tehran Stock Exchange)
}

\author{
Masomeh Mansourinia $^{1}$, Alireza Momeni ${ }^{2 *}$ \\ ${ }^{1,2}$ Department of Accounting, Payam Noor University, Tehran, Iran \\ *Corresponding Author: momeni50688@gmail.com
}

\begin{abstract}
One of the most studied variant of portfolio optimization problems is with cardinality constraints that transform classical mean-variance model from a convex quadratic programming problem into a mixed integer quadratic programming problem which brings the problem to the class of NPComplete problems. Therefore, the computational complexity is significantly increased since cardinality constraints have a direct influence on the portfolio size. In order to overcome arising computational difficulties, for solving this problem, researchers have focused on investigating efficient solution algorithms such as metaheuristic algorithms since exact techniques may be inadequate to find an optimal solution in a reasonable time and are computationally ineffective when applied to large-scale problems. In this paper, our purpose is to present an efficient solution approach based on an artificial bee colony algorithm with feasibility enforcement and infeasibility toleration procedures for solving cardinality constrained portfolio optimization problem. Computational results confirm the effectiveness of the solution methodology. In this study, the ABC-I algorithm and the ABC-II algorithm, which are the modern meta-innovative models for solving optimization problems, have been used to optimize the investment portfolio with the goal of increasing returns and reducing risk. Of the 591 companies listed on the Tehran Stock Exchange, 150 companies were selected during the period from 2014 to 2018 using a systematic elimination method with limitation as the final sample. The data from these companies were analyzed using the algorithms used in the research and their performance was compared. The results of the research indicate that the ABC-II algorithm is more efficient than $\mathrm{ABC}-\mathrm{I}$ for solving the stock portfolio optimization problem.
\end{abstract}

Keywords: Artificial Bee Colony, Portfolio Optimization, Metaheuristics, Infeasibility Toleration, ABC-I Algorithm, ABC-II Algorithm 


\section{Introduction}

Markowitz, the pioneer of modern portfolio theory (Markowitz, 1952, 1959), proposed a meanvariance model recognized as a key finding in finance that quantitatively express portfolio selection problem by treating as a quadratic optimization problem. This model assumes that the future prices of the assets can be correctly reflected by the historical prices of the market characterized by a vector of expected returns and a covariance matrix based on mean of return, standard deviation of the return and its correlation with other assets. Choosing the correct combination of assets to invest in, an investor can achieve good investment performance. Getting higher returns with the same risk rate is possible by tracing the efficient frontier, a continuous curve of mean return and risk intersections that indicates the best investment strategy.

Fundamentally, the trade-off between risk and return is sought in this model. However, due to the lack of useful real life constraints in Markowitz's original model, number of assets held in the portfolio and amount of money to be invested in each asset cannot be managed by rational investors in need of foresighted decision making in financial markets.

Although the standard efficient frontier is continuous, the shape of resultant cardinality constrained efficient frontier turns out to be discontinuous (T. J. Chang, Meade, Beasley, \& Sharaiha, 2000) and the computational complexity is significantly increased since cardinality constraints have a direct influence on the portfolio size. The classical Markowitz model as a convex quadratic programming problem that has a polynomial worst case complexity bound has been transformed into a mixed integer quadratic programming problem by adding cardinality constraints which brings the problem to the class of NP-Complete problems (Moral-Escudero, Ruiz-Torrubiano, \& Suarez, 2006). In order to overcome arising computational difficulties discussed by Fieldsend, Matatko, and Peng (2004) and Maringer and Kellerer (2003), several authors have investigated a variety of methodologies in the last two decades. Researchers have focused on the metaheuristic algorithms for solving complex constrained portfolio optimization problems since exact techniques may be inadequate to find an optimal solution in a reasonable time and are computationally ineffective when applied to largescale problems.

The purpose of this paper is to present an efficient solution approach based on an artificial bee colony (ABC) algorithm for solving CCPO problem. Artificial bee colony (ABC) algorithm, since proposed by Karaboga (2005), has been successfully applied to various combinatorial optimization problems (Can B. Kalayci \& Gupta, 2013; C. B. Kalayci, Hancilar, Gungor, \& Gupta, 2015). According to our knowledge, based on the formulation of T. J. Chang, et al. (2000), there are a few studies (A. H. L. Chen, Liang, \& Liu, 2013; A. H. L. Chen, Yun-Chia, \& Chia-Chien, 2012) in the literature that investigate the performance of $\mathrm{ABC}$ on CCPO. In general, various studies utilized a repair procedure (Anagnostopoulos \& Mamanis, 2011a, 2011b; T.-J. Chang, Yang, \& Chang, 2009; T. J. Chang, et al., 2000; Khalidji, Zeiaee, Taei, Jahed-Motlagh, \& Khaloozadeh, 2009; Khin Lwin, Qu, \& Kendall, 2014; Mashayekhi \& Omrani, 2016; Mishra, Panda, \& Majhi, 2014; Moral-Escudero, et al., 2006; Pai \& Michel, 2009; Woodside-Oriakhi, Lucas, \& Beasley, 2011) to satisfy all constraints at once that forces the solution to stay within predetermined limits at all times. This enforcement alone may prevent the algorithm to move freely in the search space resulting in a poor convergence.

\section{Literature Review}

Several studies have been done to solve the problem of optimizing portfolio constraints single solution based approaches such as SA and TS drew less attention compared to population based 
approaches such as GA and PSO. Although the nature of the problem is multi-objective, researchers mostly preferred to use single objective based approaches utilizing weighted sum approach to obtain a single objective rather than simultaneously dealing with both objectives. Evolutionary based methodologies seem to be more popular, yet swarm based methodologies are drawing more and more attention as years pass.

Among the attempts on enhancing and making Markowitz's model more practical such as limiting the number of assets to be held and lower and upper bounds on the proportion of each asset, by far the most well accepted formulation is introduced by T. J. Chang, et al. (2000) for cardinality constrained portfolio optimization (CCPO) problem. Although some authors (T. J. Chang, et al., 2000; Fernández\& Gómez, 2007) concluded that there was no one heuristic method that can outperform all others and had been proved to be superior against each other, investigating efficient solution algorithms is continued by researchers.

\section{Cardinality Constrained Portfolio Optimization Problem}

The mathematical formulation of CCPO problem based on the concept introduced by T. J. Chang, et al. (2000) is given as follows:

$\begin{array}{lll}\text { Parameters } & \begin{array}{ll}N & \text { Number of available assets } \\ \mu_{i} & \text { Expected return of asset } i \\ \sigma_{i j} & \text { Covariance between asste } i \text { and asset } j \\ \lambda & \text { Weighting parameter of variance and return }\end{array} \\ K & \text { Desired number of asstesto be hold in the portfolio } \\ \varepsilon_{i} & \text { Minimum proportion of aseet } i\end{array}$


Subject to:

$$
\begin{array}{cc}
\sum_{i=1}^{N} w_{i}=1 & \\
\sum_{i=1}^{N} z_{i}=K & \\
\varepsilon_{i} z_{i} \leq w_{i} \leq \delta_{i} z_{i} & \\
z_{i} \in\{0,1\} & i=1, \ldots, N \\
0 \leq w_{i} \leq 1, & i=1, \ldots, N \\
0 \leq \varepsilon_{i} \leq \delta_{i} \leq 1, & i=1, \ldots, N \\
& i=1, \ldots, N
\end{array}
$$

The quadratic objective function given in equation (1) seeks the best trade-offs between two conflicting objectives, maximizing return and minimizing risk. Equation (2) ensures that proportions add to one. Equation (3) guarantees that the number of assets are hold in the portfolio is exactly while Equation (4) restricts the proportion of an asset to be between predetermined values of minimum and maximum limits. Decision variable is defined in Equation (5). Equation (6) and Equation (7) defines variable domains.

Based on (Karaboga \& Akay, 2011), a general ABC algorithm is adapted to solve CCPO. The ABC algorithm starts by generating a uniformly distributed random initial population of $S N$ solutions using Equation (8) within allowed lower and upper limits.

$$
x_{i j}=x_{j}^{\text {min }}+\operatorname{rand}(0,1)\left(x_{j}^{\text {max }}-x_{j}^{\text {min }}\right) \quad i=1,2, \ldots, S N \text { and } j=1,2, \ldots, D
$$

$S N$ where denotes the number of solutions in the population and $D$ is the problem dimension (number of assets).

In the employed bee phase, a new solution is produced from the current solution with a probability depending on the modification rate ( where is a control parameter of $\mathrm{ABC}$ algorithm in the range of $[0,1]$ which restricts the modification of problem dimension. A greater modification rate leads up to a substantial alteration on the current solution while a lesser modification rate results in a minor alteration. A new solution is obtained from a previous solution located in the memory using Equation (9).

$$
v_{i j}=x_{i j}+\phi_{i j}\left(x_{i j}-x_{k j}\right) \quad k=1,2, \ldots, S N \text { and } k \neq i, \phi_{i j} \in[-1,1]
$$

where $k$ is a randomly selected index which is not equal to index $\mathrm{i}$ and $\phi_{i j}$ is a uniformly distributed random number. Thus, a solution is obtained by calculating a new position according to the current position of the selected bee and another bee's position randomly selected from the population.

In the onlooker bee phase, a selection mechanism is used to probabilistically select a better solution to be exploited by an onlooker bee. The nectar information is evaluated and a food source is probabilistically chosen in direct proportion to the probability value $P_{x i}$ associated with that nectar amount: 


$$
P_{x i}=f_{x i} / \sum_{i=1}^{S N} f_{x i}
$$

where $f_{x i}$ is the fitness value of the solution $x_{i}$. In both employed and onlooker bee phases, if bees cannot improve the solution, the corresponding limit counter value is increased, otherwise the better solution found by the bees replaces the former solution in the population.Employed and onlooker bee phases are continued until all bees complete the search process.

In the scout bee phase, another control parameter, scout production period $(s p p)$, is employed to delay exploration process. In each $s p p$ cycle, if previously calculated limit counter value exceeds the predetermined limit value (ilimit) for that particular memory, a scout bee is unleashed to randomly explore undiscovered solution space, i.e., a memory reset is provided, otherwise no scout bee moves in. Scout production process of the algorithm provides a diversification mechanism that may allow infeasible solutions to be in the population. Three phases are continued until predetermined number of iterations $(T)$ is exceeded.

\section{Enhanced Artificial Bee Colony Algorithm for CCPO}

This study thoroughly explains two attempts on solving CCPO by artificial bee colony algorithm. First attempt, utilizes the popular repair mechanism defined by T. J. Chang, et al. (2000) along with an adaptation of artificial bee colony algorithm while the second attempt, based on (Deb, 2000) and (Karaboga \& Akay, 2011), proposes an alternative approach that provides an infeasibility toleration procedure and a partial repair procedure the general $\mathrm{ABC}$ algorithm (ABC-I), the I -I method, evaluates the -I method, and selects the robot according to the objective of the function defined by equation (1), whereas the second algorithm ( ABC-II) II, the evaluation method-II. The main point of the repair mechanism is that the algorithm may move freely in space search, which causes poor convergence; therefore, an alternative repair method is provided to provide a process release release by assuring equation (3), while The difficulty constraint defined by equation (4) is examined as a repair-II repair. If the Repairs II method is used at the expense of the repair method, my assessment method is not directly applicable because it may not have a solution to the problem, therefore, an alternative assessment method is introduced. In addition to the risk, the efficiency and compatibility measures, the evaluation method-II calculate the feasibility of the solution and calculate an error value for the use of the next steps of the algorithm.

As Deb (2000) suggested, this strategy CCPO allows feasible solutions with the highest priority, less violated solutions with medium priority and highly violated solutions with the least priority. Thus, smaller constraint violation is always preferred against greater constraint violation.

The probability calculation procedure is based on Karaboga and Akay (2011) that limits the selection probabilities of violated solutions. The use of Deb's constraint handling method (Deb, 2000) alone in the algorithm, such as in GA, causes lack of diversity in the population because feasible solutions are always preferred to infeasible ones (Karaboga \& Akay, 2011). A newly obtained infeasible solution produced by the employed or onlooker bees from a previous solution in the population may join into the population if and only if the previous solution is infeasible and has a greater violation of constraints. If the previous solution is feasible, an infeasible, yet very promising solution cannot be accepted to the population. For this reason, diversification is limited and exploration may not be adequately achieved. In order to strengthen exploration and provide diversification, as a powerful mechanism of $\mathrm{ABC}$ 
algorithm, scout bees permit infeasible solutions to join the population and therefore, a search in the infeasible region is temporarily granted. Thus, a better convergence is achieved.

\section{Testing the research hypothesis}

H0: The ABC-I algorithm is more efficient in optimizing stock portfolios than the ABC-II algorithm.

H1: The ABC-I algorithm does not have much better performance than the ABC-II algorithm in optimizing stock portfolios.

\section{Research variables}

\section{Stock basket}

A basket of stocks is referred to as a combination of assets selected for investment. Stock portfolio management faces the issue of how investors can allocate their capital to different assets. Markowitz (1959) defines stock portfolio management as a process in which optimized invested weights are determined for assets that make up the stock portfolio. In the issue of portfolio selection, the purpose of allocating limited assets to assets is to profit from a viable investment strategy (Chang et al., 2000). Bayzoli-Soler et al. (2012) defined portfolios management to obtain a portfolio of portfolios with the least risk and highest returns. The investor must not only maximize returns, but must also seek to minimize risk. In fact, the risks and returns are taken into account simultaneously and the balance between risk and returns is sought.

\section{Returns}

One of the variables in this research is the average stock return of each company. Returns can be defined as investment income. In the classic model, the expected returns of the stock portfolio are equal to the linear combination of the expected return on assets in the basket of shares (Fama and Macbeth). Relationship No. (11) shows how to calculate the average stock return of each company over a period of 36 months.

$$
\mu=\frac{\sum_{i=1}^{60} r_{i}}{N}
$$

The return on equity (RIT) is calculated using relationship (12) of the Ismaili and Gnostic Studies (2005).

$$
r_{i t}=\frac{(1+\alpha) p_{1}-p_{0}+D P S-1000 \beta}{p_{0}} * 100
$$

In this research, information about the dividend yield of each share, cash flows, demands, stock prices at the beginning and end of the period, and capital at the beginning and the end of the period are also gathered using the new generation software. Relationships 13 and 14, respectively, show how to calculate the percentage of total increase in capital and the percentage of capital increase from the place of cash and demand in this research.

$$
\text { Percentage of total capital increase }=\frac{\text { End capital period }- \text { Capital of the beginning of the period }}{\text { Capital of the beginning of the period }}
$$


Percentage increase in capital from cash receipts and receivables $=\frac{\text { Increased capital from cash receipts and claims }}{\text { Capital of the beginning of the period }}$

\section{Risk}

Risk can be defined as the probability that a prediction will not occur. Another definition of risk is the likelihood of failure. As much as possible, the risk of investment failure is higher. Bradshaw et al. (2009) defined risk as a measure of what is unknown about the stock portfolio. Given this risk definition, there is a chance of increasing or decreasing the amount invested on any asset in the stock portfolio. In this study, standard deviation was used as a risk index.

\section{Research method}

The choice of research methodology depends on the purpose, nature of the subject, and its operational capabilities. This research is a descriptive-applied research field. In this research, the researcher tries to report what is there without interference or mental inference, and does not necessarily seek to discover and explain relationships, correlations, assumptions, and predictions of events, but more to focus on describing and describing situations based on Information that has only a descriptive aspect. From the dimension of the process, this research is small, that is, with objective attitudes, it collects actual data and analyzes them using relevant methods.

The statistical population of this research includes all companies accepted in the Tehran Stock Exchange during the 36-month period from 2014 to 2018, their number is based on the modern software of 591 companies.

Limiting the statistical society of the present study, among the companies accepted in the Tehran Stock Exchange, all eligible companies were selected as sample elements irrespective of the type of activity:

1) Deal on their stock does not have a long delay (more than 4 months).

2) Not part of financial investment companies.

3) The required information for research variables is available to them.

Finally, the statistical population was selected using a systematic elimination method and according to the conditions and restrictions imposed, 150 companies were selected as sample during the 36 month period ended March 2018.

In this research, a hybrid approach has been used to select stock portfolios. The following Fig.1, shows the implementation framework: 


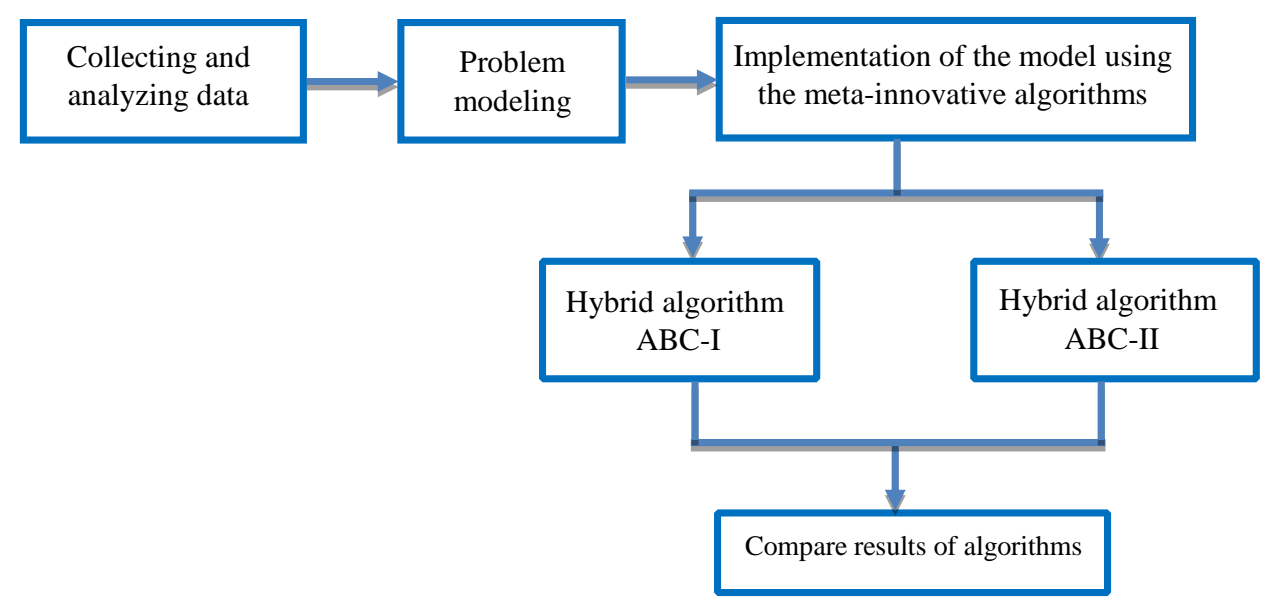

Figure 1. The framework for research implementation

\subsection{Descriptive statistics}

Regarding the limitations on the statistical society, the selected sample, regardless of the type of industry and industry, has been selected among the companies admitted to the Tehran Stock Exchange, which covers various industries. The chart below shows the frequency of selected companies in terms of industry.

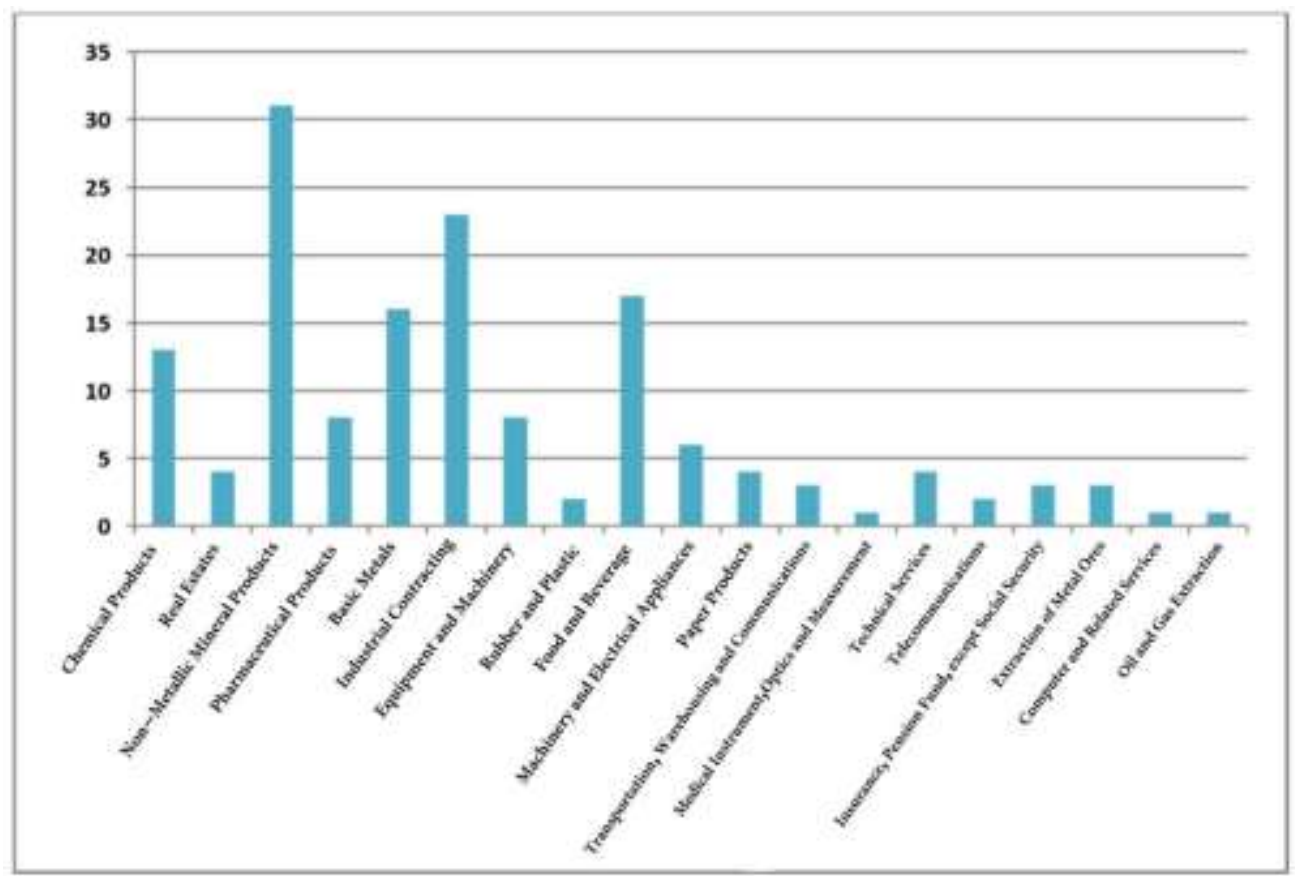

Figure 2. Selected sample among companies accepted in Tehran Stock Exchange 
As can be seen in Fig. 2, the sample of 19 companies is from the industry in the Tehran Stock Exchange. The largest number of sample companies belongs to the non-metallic mineral products industry. Table 1 examines the values of the numerical indices of the research variables.

Table 1. Descriptive statistics of research variables

\begin{tabular}{cccccc}
\hline Variable Name & Maximum & Minimum & $\begin{array}{c}\text { Standard } \\
\text { Deviation }\end{array}$ & Middle & Average \\
\hline Average stock return & 1.67 & -0.506 & 0.222 & 0.383 & 0.463 \\
\hline Covariance between Stock companies & 9.41 & $0 / 0029$ & 0.944 & 0.48 & 0.94 \\
\hline
\end{tabular}

According to the information in Table 1, the average return on equity is between -0.506 and 1.67 , with an average of 0.463 The covariance between the shares of the companies varies from 0.0029 to 9.41 and is on average about 0.94 .

\subsection{Stock bases based on the Markovitz-Variance model}

In this research, the possibility of forming an optimal stock basket was tested using research data. To this end, the risk chart and expected returns of stock exchanges are based on the Markovitz averagevariance model. The limitations are as follows:

1. The weight of each company's stock is greater than or equal to zero.

2. The total weight of the stock of 150 sample companies is equal to one.

3. Expected stock returns from 0.1 to 1 .

Figure 3, shows Stock Stocks based on the Markovitz-Variance Model. The horizontal and vertical axis of this chart, respectively, indicates the stock portfolio risk and the expected returns of the stock basket. Each point on this chart represents a basket with a certain expected return and a distinct risk. In this chart, based on 10 times the EXCEL 2010 version, the stock basket has reached 10 points.

As shown in Fig.3, using the data from this study, an equity basket can be optimized and efficient, so that the risk increases with increasing expected returns. In addition, based on the outputs obtained from the analysis, the expected range of the stock basket varies from 0.1 to 1 with a 0.1 step and creates a risk of between 0.001 and 0.04 .

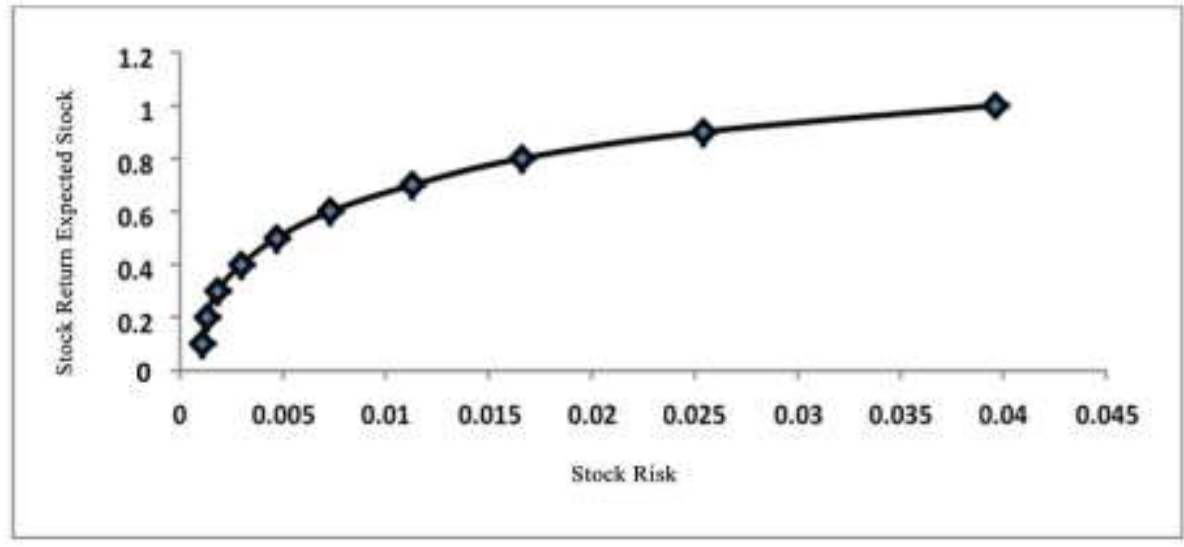

Figure 3. Stocks based on the average-variance model

\subsection{ABC-I algorithm and ABC-II algorithm}


The benchmark for the ABC-I and ABC-II algorithms is set by test, error, and appropriate timing to solve the problem of this research. Table 2, shows the criteria for the indicators of this algorithm.

Table 2. Benchmarking of the ABC-I and ABC-II algorithms

\begin{tabular}{cccc}
\hline Indicator & Criterion & Indicator & Criterion \\
\hline $\begin{array}{c}\text { Primary Worker Bee } \\
\text { Population }\end{array}$ & 10 & Mutation rate & 0.001 \\
\hline $\begin{array}{c}\text { Primary Observer Bee } \\
\text { Population }\end{array}$ & 10 & Intersection rate & 0.2 \\
\hline Maximum number of replays & 500 & Risk factor & 0.2 \\
\hline
\end{tabular}

The benchmark for the ABC-II algorithm is set by test and error and in a suitable state for solving the problem of this research. Table 3, shows the benchmark for the indicators of this algorithm.

Table 3. Benchmarking of the ABC-II algorithm

\begin{tabular}{cccc}
\hline Indicator & Criterion & Indicator & Criterion \\
\hline Initial probability vector & 0.51 & Mutation rate & 0.005 \\
Lite size $(\mathrm{M})$ & 5 & Intersection rate & 0.2 \\
Maximum number of replays & 500 & Risk factor & 0.2 \\
Learning rate & 0.1 & Negative learning rate & 0.075 \\
Rate probability mutation rate & 0.5 & Likelihood of mutation & 0.5 \\
\hline
\end{tabular}

\subsection{Comparison of used algorithms in research}

As shown in Fig.4, shows the effective boundary of the ABC-I algorithm and the ABC-II algorithm for the 10 stock portfolios obtained by running the Visual Studio 2010 software and the $\mathrm{C}$ \# programming language. Any point on this figure represents a basket with risk and efficiency.

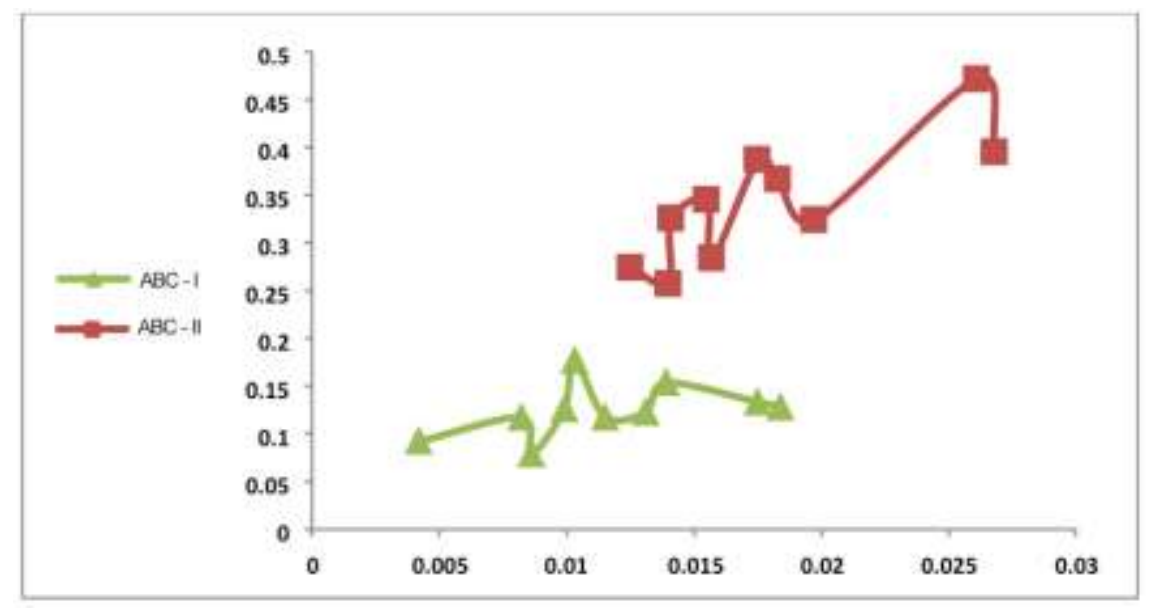

Figure 4. Comparison of the effective boundary of algorithms used in research

Since the risk aversion coefficient of the investor in this research is 0.2 (investor venture), higher returns are more important than risk reduction. As a result, the higher the risk-return slope is, the better the situation. As shown in Fig.4, the slope of the ABC-II algorithm is more than the ABC-I algorithm. 
The points of the ABC-II algorithm at the level of risk equalization are more efficient than the ABC-I algorithm. Therefore, the ABC-II algorithm is more favorable than the ABC-I algorithm, so the ABCII algorithm, which is more favorable than $\mathrm{ABC}-\mathrm{I}$, is an efficient algorithm for optimizing the investment basket.

The ability of ABC-I and ABC-II algorithms was evaluated in providing an optimal stock portfolio of two dimensions of stock portfolio formation and the design of an effective portfolio of stock portfolios. Table 4, shows the ability of these algorithms to provide optimal stock baskets.

Table 4. Expected Output Results for Applied Algorithms

\begin{tabular}{c|c|c|c|c}
\hline \multirow{2}{*}{$\begin{array}{c}\text { Stock } \\
\text { Basket }\end{array}$} & \multicolumn{2}{|c|}{ ABC-I Algorithm } & \multicolumn{2}{c}{ ABC-II Algorithm } \\
\cline { 2 - 5 } & Risk & Expected Output & Risk & Expected Output \\
\hline 1 & 0.0042 & 0.0919 & 0.116 & 0.266 \\
\hline 2 & 0.0082 & 0.117 & 0.0130 & 0.245 \\
\hline 3 & 0.0086 & 0.079 & 0.0137 & 0.242 \\
\hline 4 & 0.0092 & 0.1261 & 0.0138 & 0.275 \\
\hline 5 & 0.0130 & 0.1772 & 0.0162 & 0.312 \\
\hline 6 & 0.0115 & 0.1175 & 0.0186 & 0.338 \\
\hline 7 & 0.0113 & 0.1229 & 0.0196 & 0.394 \\
\hline 8 & 0.0139 & 0.1534 & 0.0197 & 0.394 \\
\hline 9 & 0.0175 & 0.1332 & 0.0201 & 0.353 \\
\hline 10 & 0.0184 & 0.1282 & 0.0209 & 0.395 \\
\hline
\end{tabular}

According to the information in

Table 5, using the ABC-I algorithm of the ABC-II algorithm, an optimized stock basket can be created so that the expected yield increases with increasing risk.

Table 5. Comparison of the average returns of the stock baskets optimized by the ABC-I and ABC-II algorithms

\begin{tabular}{c|c|c|c|c|c|c|c}
\hline \multirow{2}{*}{ Variable } & Algorithm & \multirow{2}{*}{$\begin{array}{c}\text { Stock } \\
\text { Stocks }\end{array}$} & \multirow{2}{*}{ Average } & \multicolumn{2}{|c|}{$\begin{array}{c}\text { Equivalence Test for } \\
\text { Variances }\end{array}$} & $\begin{array}{c}\text { Test of Mean } \\
\text { Comparison }\end{array}$ & $\begin{array}{c}\text { Test for the } \\
\text { Comparison of } \\
\text { Variances }\end{array}$ \\
\cline { 5 - 8 } & & & F & Sig & T & T \\
\hline \multirow{2}{*}{$\begin{array}{c}\text { Average } \\
\text { Return }\end{array}$} & ABC-I & 10 & 0.1246 & \multirow{2}{*}{0.2002} & 0.2481 & 0.0252 & 0.47116 \\
\cline { 8 - 8 } & ABC-II & 10 & 0.3425 & & 0.4723 \\
\hline
\end{tabular}

According to Table 5, which is presented based on the expected results of the expected returns of the two algorithms, the first co-variance test was carried out. The F statistic and its significant level were 0.2002 and 0.2481 , respectively, with a significant level of greater than 0.05 , The variance of the two algorithms is equal. Also, according to the results of the comparison of the variances of the two algorithms by the T-statistic, the variance of the two algorithms is equal, the variance of the ABC-I algorithm is 0.47116 and the ABC-II variance is 0.4723 .

Also, the t-test statistic obtained at 0.0252 is not within $95 \%$ confidence interval $(\alpha=0.05)$, so the assumption $\mathrm{H} 0$ is rejected, and we can say that optimization of stock portfolio by ABC-II algorithm is more efficient It has the ABC-I algorithm.

\section{Conclusion}


As stated, investment is one of the essential and elements of the country's economic growth and development process and is an important factor in investing the investor's attention to risk and return. Investors are trying to invest their finances in the place that has the highest returns and the least risk have. In order to answer the first question, the problem of choosing the optimal stock basket and determining the effective boundary using the meta-initiative algorithm were considered. In order to answer the second research question, the expected risk and expected returns of stock portfolios of the algorithms used in the research were compared. The expected average probability of the ABC-II algorithm is 0.3425 and the average risk of the ABC-II algorithm is 0.01802 . These values are expected with the average expected yield of ABC-I algorithm (0.1246) and the average risk of the ABC-I algorithm (0.01156) are comparable. Given the results, it is clear that the proposed algorithms have a good performance for solving the stock portfolio optimization problem. The results of the research show that using the ABC-II algorithm, we can construct the optimal stock basket with a higher accuracy and efficiency, such that the slope of the risk-return diagram is greater than that of the ABC-I algorithm.

\section{REFERENCE}

Elahi Morteza, Yousefi Mohsen, Zare Mehrjerdi Yahya, (2015). "Optimization of the stock portfolio with the mean value of the variance and using the search algorithm for hunting". Financial Research Faculty of Management, University of Tehran. No. 16, pp. 56-37.

Amir Hosseini Zahra, Ghobadi Masoumeh, (2016). "Evaluation and selection of stock portfolios using fuzzy theory and multi-criteria decision making". Journal of Financial Engineering and Management of Securities, No. 27, pp. 16-1.

Khosh Tinat Mohsen, Esmaili Shapour, (2005). "The Relationship Between Quality of Profit and Stock Returns". Empirical Financial Accounting Studies, No. 12 \& 13, pp. 56-27.

Raei Reza, Ali Beygi Hedayat, (2010). "Stocks portfolio optimization using particle motion method". Financial Research Faculty of Management, University of Tehran, No. 29, pp. 40-21.

Zamani Hasan, Afsar Amir, Saghafi Seyed Vahid, Bayat Elham, (2013). "Expert system of stock price prediction and portfolio optimization using fuzzy neural networks, fuzzy modeling and genetic algorithm". Financial Engineering and Securities Management, No. 21, pp. 130-107.

Shahmohammadi Mohsen, Emami Meybodi Lili, Zare Mehrjerdi, Yahya (2012). "Intelligent combination algorithm based on fuzzy model of mean variance-skewness for selection of portfolios". International Journal of Industrial Engineering and Production Management, No. 4, pp. 458-448.

Quarterly Journal of Stock Exchange, No. 18, pp. 59-39.

Ghasemi Hamid Reza, Najafi Amir Abbas, (2012). "Presentation of weed optimization algorithm for solving investment portfolio selection problem". Eighth International Industrial Engineering Conference.

Tuba, M., \& Bacanin, N. (2014b). Upgraded firefly algorithm for portfolio optimization problem. In Proceedings - UKSim-AMSS 16th International Conference on Computer Modelling and Simulation, UKSim 2014 (pp. 113-118). 
Baixauli-Soler J. S., Alfaro-Cid, E., Fernandez-Blanco, M. O. (2012). "A naïve approach to speed up portfolio optimization problem using a multiobjective genetic algorithm". Investigaciones Europeas de Dirección y Economía de la Empresa, Vol.18, pp. 126-131.

Bermúdez, J. D., Segura, J. V., Vercher, E. (2012). "A multi-objective genetic algorithm for cardinality constrained fuzzy portfolio selection". Fuzzy Sets and Systems, Vol. 188, pp. 16-26.

Chang, T. J., Meade, N., Beasley, J. E., Sharaiha, Y. M. (2000). "Heuristics for cardinality constrained portfolio optimisation". Computers \& Operations Research, Vol. 27, pp. 1271-1302.

Chang, T. J., Yang, S. C., Chang, K. J. (2009). "Portfolio optimization problems indifferent risk measures using genetic algorithm". Expert Systems with Applications, Vol. 36, pp. 10529-10537.

Chen, W. (2015). "Artificial bee colony algorithm for constrained possibilistic portfolio optimization problem". Physica A: Statistical Mechanics and its Applications, Vol. 429, pp. 125-139.

Chiam, S. C., Tan, K. C., Mamun, A. A. (2009). "A memetic model of evolutionary PSO for computational finance applications". Expert Systems with Applications, Vol. 36, pp. 3695-3711. Cura, T. (2009). "Particle swarm optimization approach to portfolio optimization". Nonlinear Analysis: Real World Applications, Vol. 10, pp. 2396-2406.

Fernández, A., Gómez, S. (2007). "Portfolio selection using neural networks". Computers \& Operations Research, Vol. 34, pp. 1177-119.

Golmakani, H. R., Jalilipour Alishah, E. (2008). "Portfolio selection using an artificial immune system". In Information Reuse and Integration, IEEE International Conference on 2008, pp. 28-33.

Lwin, K., Qu, R. (2013). "A hybrid algorithm for constrained portfolio selection problems". Applied intelligence, Vol. 39, pp. 251-266.

Mansini, R., Speranza, M. G. (1999). "Heuristic algorithms for the portfolio selection problem with minimum transaction lots". European Journal of Operational Research, Vol. 114, pp. 219-233.

Markowitz, H. (1952). "Portfolio selection". The journal of finance, Vol. 7, pp. 7791.

Markowitz, H. (1959). "Portfolio selection: efficient diversification of investments". Cowies Foundation Monograph.

Markowitz, H. (2014). "Mean-variance approximations to expected utility". European Journal of Operational Research, Vol. 234, pp. 346-355.

Storn, R., Price, K. (1997). "Differential evolution - a simple and efficient heuristic for global optimization over continuous spaces". Journal of Global Optimization, Vol. 11, pp. 341-359

Yu, M., Takahashi, S., Inoue, H., Wang, S. (2010). "Dynamic portfolio optimization with risk control for absolute deviation model”. European Journal of Operational Research, Vol. 201, pp. 349-364.

Anagnostopoulos, K. P., \& Mamanis, G. (2011a). The mean-variance cardinality constrained portfolio optimization problem: An experimental evaluation of five multiobjective evolutionary algorithms. Expert Systems with Applications, 38, 14208-14217.

Anagnostopoulos, K. P., \& Mamanis, G. (2011b). Multiobjective evolutionary algorithms for complex portfolio optimization problems. Computational Management Science, 8, 259-279. 
Baykasoğlu, A., Avci, M. G., \& Burcin Özsoydan, F. (2016). Erratum to "A GRASP basedsolution approach to solve cardinality constrained portfolio optimization problems"[Comput. Indus. Eng. 90 (2015) 339-351]. Computers \& Industrial Engineering, 96, 249-250.

Baykasoğlu, A., Yunusoglu, M. G., \& Burcin Özsoydan, F. (2015). A GRASP based solution approach to solve cardinality constrained portfolio optimization problems. Computers \& Industrial Engineering, 90, 339-351.

Beasley, J. E. (1990). OR-Library. In (Vol. 2016).

Branke, J., Scheckenbach, B., Stein, M., Deb, K., \& Schmeck, H. (2009). Portfolio optimization with an envelope-based multi-objective evolutionary algorithm. European Journal of Operational Research, $199,684-693$.

Cesarone, F., Scozzari, A., \& Tardella, F. (2013). A new method for mean-variance portfoliooptimization with cardinality constraints. Annals of Operations Research, 205, 213- 234.

Chen, A. H. L., Liang, Y. C., \& Liu, C. C. (2013). Portfolio optimization using improved artificial bee colony approach. In Proceedings of the 2013 IEEE Conference on Computational Intelligence for Financial Engineering and Economics, CIFEr 2013 - 2013 IEEE Symposium Series on Computational Intelligence, SSCI 2013 (pp. 60-67).

Cui, T., Cheng, S., \& Bai, R. (2014). A combinatorial algorithm for the cardinality constrained portfolio optimization problem. In Proceedings of the 2014 IEEE Congress on Evolutionary Computation, CEC 2014 (pp. 491-498).

Deb, K. (2000). An efficient constraint handling method for genetic algorithms. Computer Methods in Applied Mechanics and Engineering, 186, 311-338.

Deng, G.-F., Lin, W.-T., \& Lo, C.-C. (2012). Markowitz-based portfolio selection with cardinality constraints using improved particle swarm optimization. Expert Systems with Applications, 39, 45584566.

Fieldsend, J. E., Matatko, J., \& Peng, M. (2004). Cardinality Constrained Portfolio Optimisation. In Z. R. Yang, H. Yin \& R. M. Everson (Eds.), Intelligent Data Engineering and Automated Learning IDEAL 2004: 5th International Conference, Exeter, UK. August 25-27, 2004. Proceedings (pp. 788793). Berlin, Heidelberg: Springer Berlin Heidelberg.

Fogarasi, N., \& Levendovszky, J. (2013). Sparse, mean reverting portfolio selection using simulated annealing. Algorithmic Finance, 2, 197-211.

Ge, M. (2015). Artificial bee colony algorithm for portfolio optimization. In 5th International Conference on Intelligent Control and Information Processing, ICICIP 2014 - Proceedings (pp. 449453).

Jin, Y., Qu, R., \& Atkin, J. (2015). A population-based incremental learning method for constrained portfolio optimisation. In Proceedings - 16th International Symposium on Symbolic and Numeric Algorithms for Scientific Computing, SYNASC 2014 (pp. 212- 219).

Kalayci, C. B., Hancilar, A., Gungor, A., \& Gupta, S. M. (2015). Multi-objective fuzzy disassembly line balancing using a hybrid discrete artificial bee colony algorithm. Journal of Manufacturing Systems, 37, 672-682.

Kao, Y., \& Cheng, H.-T. (2013). Bacterial Foraging Optimization Approach to Portfolio Optimization. Computational Economics, 42, 453-470. 
Karaboga, D. (2005). An idea based on Honey Bee Swarm for Numerical Optimization. In. Kayseri: Erciyes University, Engineering Faculty, Computer Engineering Department.

Karaboga, D., \& Akay, B. (2011). A modified Artificial Bee Colony (ABC) algorithm for constrained optimization problems. Applied Soft Computing, 11, 3021-3031.

Khalidji, M., Zeiaee, M., Taei, A., Jahed-Motlagh, M. R., \& Khaloozadeh, H. (2009).

Liagkouras, K., \& Metaxiotis, K. (2014). A new Probe Guided Mutation operator and its application for solving the cardinality constrained portfolio optimization problem. Expert Systems with Applications, 41, 6274-6290.

Lwin, K., Qu, R., \& Kendall, G. (2014). A learning-guided multi-objective evolutionary algorithm for constrained portfolio optimization. Applied Soft Computing, 24, 757- 772.

Mishra, S. K., Panda, G., \& Majhi, R. (2014). Constrained portfolio asset selection using multiobjective bacteria foraging optimization. Operational Research, 14, 113-145.

Moral-Escudero, R., Ruiz-Torrubiano, R., \& Suarez, A. (2006). Selection of Optimal Investment Portfolios with Cardinality Constraints. In Evolutionary Computation, 2006. CEC 2006. IEEE Congress on (pp. 2382-2388).

Ni, Q., Yin, X., Tian, K., \& Zhai, Y. (2016). Particle swarm optimization with dynamic random population topology strategies for a generalized portfolio selection problem. Natural Computing, 1-14.

Pai, G. A. V., \& Michel, T. (2009). Evolutionary optimization of constrained k-means clustered assets for diversification in small portfolios. IEEE Transactions on Evolutionary Computation, 13, 1030-1053.

Suthiwong, D., \& Sodanil, M. (2016). Cardinality-constrained Portfolio optimization using an improved quick Artificial Bee Colony Algorithm. In 2016 International Computer Science and Engineering Conference (ICSEC) (pp. 1-4).

Thomaidis, N. S. (2010). Active Portfolio Management from a Fuzzy Multi-objective Programming Perspective. In C. Chio, A. Brabazon, G. A. Caro, M. Ebner, M. Farooq, A. Fink, J. Grahl, G. Vercher, E., \& Bermúdez, J. D. (2015). Portfolio optimization using a credibility meanabsolute semi-deviation model. Expert Systems with Applications, 42, 7121-7131.

Wang, Z., Liu, S., \& Kong, X. (2011). An improved artificial bee colony algorithm for portfolio optimization Problem. International Journal of Advancements in Computing Technology, 3, 67-74.

Woodside-Oriakhi, M., Lucas, C., \& Beasley, J. E. (2011). Heuristic algorithms for the cardinality constrained efficient frontier. European Journal of Operational Research, 213, 538-550.

Yin, X., Ni, Q., \& Zhai, Y. (2015). A novel particle swarm optimization for portfolio optimization based on random population topology strategies. In Lecture Notes in Computer Science (including subseries Lecture Notes in Artificial Intelligence and Lecture Notes in Bioinformatics) (Vol. 9140, pp. 164-175).

Tuba, M., \& Bacanin, N. (2014b). Upgraded firefly algorithm for portfolio optimization problem. In Proceedings - UKSim-AMSS 16th International Conference on Computer Modelling and Simulation, UKSim 2014 (pp. 113-118). 